Monatsschrift für Geburtshülfe u. Gynäkologie 1909;30:367-372

\title{
Max Runge †
}

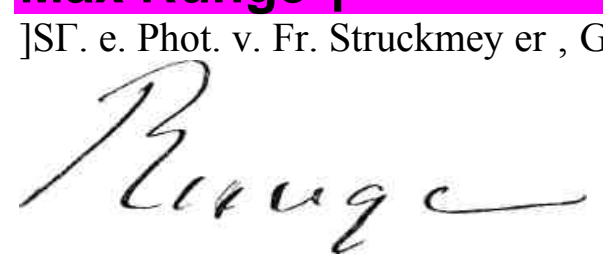

Wieder hat ein grausames Geschick eine Lücke in unsere Reihen gerissen: Runge, den wir ebenso wie Pfannenstiel in den Strassburger Tagen frisch und anscheinend in besserer Gesundheit als früher unter uns sahen, er ist jäh dahingerafft! Bald nach seiner Rückkehr nach Göttingen meldeten sich die bedrohlichen Symptome einer schweren Nierenerkrankung. Von seinem intimsten Jugend-freund anscheinend glücklich operiert, erlag er im Laufe der ersten Woohe naoh der Operation am 17. August einer Darmlähmung. In 368

Max Runge $\uparrow$.

der entfernten Niere sass ein walnussgrosses Hypernephrom. - Als wir vor 8 Jahren gemeinsam nach Giessen fuhren, hatte Runge sich selbst ein frühes Ende an einem Neoplasma prognostiziert: das werde ihm beschieden sein wie einer Reihe seiner Göttinger Kollegen! Heinrich Max Runge war am 2. September 1849 in Stettin als Sohn eines Arztes geboren. 1869 bezog er die Universität Jena. Von bier ging er ins Feld; an Ruhr erkrankt, kehrte er aus der Zernierungsarmee vor Metz zurück. Nach dem er dann in Bonn und Strassburg seine Studien fortgesetzt, bestand er 1874 in Strassburg das Staatsexamen, promovirte mit der an Gusserows Material verfassten Dissertation über Messungen der Kopf-Steiss-länge nach Ahlfeld. Er war 6 Monate Assistent bei Leyden, um sich dann vollständig Gusseroiv anzuschliessen. Diesem folgte er 1878 nach Berlin. 1879 habilitiert, wurde Runge 1883 als Ordinarius nach Dorpat berufen, 1888 nach Göttingen. Hier entfaltete er dann mit vollstem Erfolg sein Organisationstalent; in einem hervorragend schönen Neubau, der von dem preussischenMedizinal-ministerium als Modell bezeichnet wird fur seine Frauenkliniken, schuf Runge eine mustergültige Unterrichtsanstalt, mit reichem geburtshülflichem und gynäkologischem Material. Er wurde für die Studenten eine der ersten Anziehungskräfte seiner Fakultät, der Consiliarius weit über die Grenzen seiner Provinz hinaus.

Runges nächste Interessen galten der Geburtshülfe, für welche sich ihm in Strassburg und in der Berliner Charité reichliches Beobachtungsmaterial bot.

Erst später floss ihm das gynäkologische Material reichlicher zu: er erwies sich als ein ausgezeichneter Diagnostiker und Operateur. -- Mit Vorliebe ging er chemisch-biologischen Unter-suchungen nach; mehrfache kleine Aufsätze in Fachzeitschriften sind die sehr beachtenswerten, dauernd bedeutungsvollen Früchte dieser Tätigkeit. Nachhaltigeres Aufsehen erregte seine Darstellung der Krankheiten der ersten Lebenstage, welche, in 2. Auflage erschienen, auch heute noch eine fundamentale Bedeutung hat. Runge war streng kritisch, auch an sich selbst. Seine Publi-kationen folgten sich dementsprechend nur in längeren Zwischen- 
räumen. Dafür waren aber besonders seine Lehrbücher klassische Typen der Darstellung des Lehrstoffes in prägnantester Form. Seine Geburtshülfe hat er eben erst, 1909, in 8. Auflage in modernster Ausstattung und zeitentsprechender Umarbeitung vollendet, die Gynäkologie 1907 in 3. Auflage.

Obwohl selbst als Hebammenlehrer nur in seiner Assistenten-zeit tätig, wurde Runge von der Kommission für den Entwurf eines

Max Runge $\dagger$.

369

neuen preussischen Hebammenbuches mit dieser Aufgabe betraut. In seiner knappen, klaren Darstellung hat er ein vorzügliches Buch geschrieben, das besonders dann zur vollen Anerkennung kommen wird, wenn die heute allgemein betätigten Bestrebungen zur strengeren Auswahl eines intelligenteren, besser vorgebildeten Schülerinnenmaterials ihre Trüchte getragen haben. Ganz besonders aber muss jeder Autor die vornehme und geschickte Art anerkennen, mit der Runge es verstanden hat, die Ergebnisse der Beratungen dieser vielköpfigen Kommission zu einem einheitlichen Ganzen zu verschmelzen.

Mit der ihm eigenen besonderer Schärfe hat Runge in der Frauenfrage ablehnend und abmahnend Stellung genommen. Seine Streitschrift ist in fünf Auflagen verbreitet.

Nur selten trat Runge auf Kongressen in unsere Mitte; wo er sprach, fand er schnell die voile Aufmerksamkeit der Anwesenden; was er sprach, war nachhaltig, bedeutungsvoll. So trat er auf dem ersten Gynäkologenkongress in München 1886 für Gusserow als Referent über die Therapie des Puerperalfiebers ein. Die von ihm damals empfohlene Alkoholbehandlung konnte er auch 1909, in der Diskussion in Strassburg, wieder als bewährt und angesichts der klar zutage liegenden Zweifel in Bezug auf die Therapie - als auch heute noch sehr beachtenswert bezeichnen.

Erst in diesem Jahre hat Runge der Bedeutung der kollegialen Vereinigungen eine weitere Konzession gemacht, indem er sich der Nordwestdeutschen Gynäkologen-Gesellschaft in Hamburg anschloss und zu ihrer nachhaltig en Förderung bereit erklärte.

Runges Grösse lag in seiner organisatorischen und seiner lehr-amtlichen Tätigkeit. Wusste er als Leiter der Klinik stets genati, was er wollte und was er erreichen konnte, so war er als Lehrer von fesselnder, anregender Vortragskunst, klar und prägnant im Ausdruck. Ein Mann von unermüdlicher Arbeitskraft und freudiger Diensterfüllung, verlangte er allerdings unerbittlich das gleiche von seinen Assistenten und Schülern. Im Kreis seiner Kollegen wegen der Bestimmtheit seiner Ziele und der Lauterkeit seines Charakters, als eigenartige Persönlichkeit hochgeachtet, war er in äusserem Verkehr von bestrickender Liebenswürdigkeit. Das empfanden vor alien seine Klienten, die ihm mit dankbarer Ver-ehrung ergeben waren.

Nur einem engen Kreis von Freunden erschloss sich Runges ganzes Wesen. Er fand sein Glück am eigenen Herd: seine Gattin hat ihm 10 Kinder geschenkt, den ältesten Sohn sah der Vater noch als angehenden Psychiater, die älteste Tochter als glück-

370

Max Runge $\uparrow$.

liche Ehefrau und Mutter zweier hoffnungsvoller Sprösslinge. Runge war überall als Mann und Gelehrter von hoher Intelligenz und Vornehmheit des Charakters hochgeschätzt, als liebevoller, vorsorglicher Arzt bei seinen Pfleglingen aus alien Kreisen verehrt. Wir aber betrauern in ihm einen unserer Besten, dessen Andenken auf die kommenden Generationen als das eines streng wissenschaft- 
lichen Torschers, begnadeten Lehrers und ausgezeichneten Arztes

übergeht! A. Martin. A. v. Rosthorn.

Max Runges literarische Arbeiten:

Über Messung der Kopf-Steisslänge nach Ahlfeld. Diss.-inaug. Strass-

burg 1874. Der Übergang der Salicylsäure und des Jodkaliums in das Fruchtwasser.

(Strassburg.) Centralbl. f. Gyn. 1877. Bd. I. S. 75. Untersuchungen über den Einfluss der gesteigerten mütterlichen Temperatur

in der Schwangerschaft auf das Leben der Frucht. Arch. f. Gyn.

1877. Bd. XII. S. 16. Die Wirkung hoher und niedriger Teinperaturen auf den Uterus des

Kaninchens und des Menschen. Arch. f. Gyn. 1878. Bd. XIII, S. 123. Bericht über die

Leistungen der unter der Direktion des Prof. Gusserow

stehenden geburtshülflich gynäkologischen Klinik zu Strassburg i. E.

Prager Vierteljahrsschrift. 1878. Bd. CXXXIII. Sauerstoffmangel und Ivohlensäure-Ueberschuss des Blutes in ihrer Be-

ziehung zum schwangeren und nichtschwangeren Uterus. Zeitschr.

f. Geb. u. Gyn. 1879. Bd. IV. Über den Einfluss des schwefelsauren Chinins auf den fötalen Organismus.

Zentralbl. f. Gyn. 1880. Bd. IV. No. 3. S. 49. (Berlin.) Die akuten Infektionskrankheiten in ätiologischer Beziehung zur Schwanger-

schaftsunterbrechung. Sammlung klin. Vortr. 1880. No. 177. Fall von ausgedehnter

myxomatöser Entartung der Placenta. Zentralbl. í.

Gyn. 1880. Bd. IV. S. 319. (Vortrag in der geburtshülflich-

gynäkologischen Gesellschaft zu Berlin.) Bemerkungen über eine Puerperalfieberepidemie in der geburtshüflichen

Abteilung der Charité. Zeitschr. f. Geb. u. Gyn. 1880. Bd. V. S. 195. Die Leitung der

Nachgeburtsperiode. Berl. klin. Wochenschr. 1880. No. 44. Über Nabelerkrankung und

Nabelverband. Zeitschr. f. Geb. u. Gyn. 1881.

Bd. VI. S. 64. Fall von Bauchblasengenitalspalte. Charité-Annalen. 1881. S. 648. Zur Frage nach der Ursache des ersten Atemzuges des Neugeborenen.

Zeitschr. f. Geb. u. Gyn. 1881. Bd. VI. H. 2. Bemerkung zu dem Aufsatz von H. Fehling: ,,Zur

Frage der zweck-

mässigsten Behandlung der Nachgeburtszeit". Zentralbl. f. Gyn.

1881. Bd. IV. No. 26. S. 616. Anatomische Befunde bei Neugeborenen. Aus Gusserows Klinik.

Charité-

Annalen. 1882. VII. S. 714. Luft in den Lungen totgeborener Kinder. Berl. klin. Wochenschr.

1882.

No. 11,

Max Runge T.

371

Die Veränderungen der brechenden Medien des Auges bei macerierten

Früchten. Berl. klin. Wochenschr. 1882. No. 34. Die therapeutische Anwendung des heissen

Wassers in der Geburtshülfe

und Gynäkologie. Berl. klin. Wochenschr. 1883. No. 2. Uterusreize und Uterusbewegung.

Zentralbl. f. Gyn. 1883. No. 11. S. 329.

(Vortrag in der Gesellschaft für Geb. u. Gyn. zu Berlin.) Über die Berechtigung des

Kaiserschnittes an der Sterbenden und der mit 
ihm konkurrierenden Entbindungsverfahren. Zeitschr. f. Geb. u. Gyn.

1883. Bd. IX. H. 2. Die Geburtshülfe und Gynäkologie, ein Zweig der allgemeinen Medizin. Antrittsrede, Dorpat. 1884. Deutsche med. Wochenschr. 1884. No. 12. Mitteilung über die intrauterine Ubertragbarkeit des Erisypelas. (Dorpat.)

Zentralbl. f. Gyn. 1884. S. 760. No. 48. Die Stichhaltigkeit der Lungenprobe. Eulenbergs Vierteljahrsschrift f.

gerichtl. Med. u. ö. Sanitätswesen. N. F. 1885. Bd. XLП. H. 1. Kritisches und Experimentelles zur Lehre von der Gefährlichkeit des Fiebers

in der Schwangerschaft und im Wochenbett. Arch. f. Gyn. 1885.

Bd. XXV. H. 1. Bericht über einige bemerkenswerte Laparotomien. St. Petersburger

Wochenschr. 1885. No. 51. Die Krankheiten der ersten Lebenstage. I. Aufl. Stuttgart. F. Enke. 1885.

II. Aufl. 1893. Ein Fall von Tuboovarialcyste. Arch. f. Gyn. 1886 Bd. XXVI.. H. 1. Über

Allgemeinbehandlung der Wochenbettserkrankungen. Verhandlg. d.

1. Versammlg. d. deutsch. Gesellschaft f. Gyn. inMünchen. 17-1.9. VI.

1886. Zentralbl. f. Gyn. 1886. S. 460.

Über die Behandlung der puerperalen Sepsis. Sammhmg klin. Vortr. 1886.

No. 287. (Zusammenfassung f. d. Vortrag.) Kasuistik aus der Dorpater Frauenklinik. St.

Peterburger Wochenschr.

1887. No. 19-20.

Fall von glaudulären Ovarialcystomen mit gelatinösem Inhalt und peri-tonealen Metastasen.

Zentralbl. f. Gyn. 1887. No. 15.

Vor Blasensprung abgestorbene frühreife Frucht. St. Petersburger med. Wochenschr. 1887. S.

168.

Allgemeinbehandlung der puerperalen Sepsis. Arch. f. Gyn. 4. Mitteilung

1888. Bd. XXXIII. H. 1. (Göttingen.)

Über die Behandlung der puerperalen Sepsis. Deutsche med. Wochenschr.

1889. No. 1. (Vortrag in der Göttinger med. Gesellschaft.)

Zur Therapie der Untersuchungen. Arch. f. Gyn. 1889. Bd. XXXIV. H. 3. Die Behandlung der

Wehenschwäche unter der Geburt. Therap. Monats-

hefte. 1890. Mitteilungen aus der Göttinger Frauenklinik. Arch. f. Gyn. 1891. Bd. 41.

S. 86. Lehrbuch der Geburtshülfe. I. Aufl. 1891. VIII. Aufl. 1909. Eine kurze Berichtigung (betr.

Lehrbuch). Gyn. Zentralbl. 1892. S. 116. 120 Laparotomien aus der Göttinger Frauenklinik.

Therap. Monatshefte.

Dez. 1893. Die Ursachen der Lungenatmung des Neugeborenen. Arch. f. Gyn. H. 3.

Bd. 46. 1894.

372

Druckfehler-Berichtigung.

Der erste Schrei und der erste Atemzug. Berliner klin. Wochenschr. 1895.

No. 5. Exstirpation einer Wandermilz mit Achsendrehung des Stiels. Berliner

klin. Wochenschr. 1895. No. 16. Stumpfbehandlung nach sapravaginaler Amputation des Uterus.

Zentralbl.

f. Gyn. 1895. S. 1289. Ein neuer Fall von bösartigem Tumor der Chorionzotten. Arch. f. Gyn. 1896. Bd. LI. Die sogenannten Hülfsursachen des ersten Atemzuges und die intrauterine Atmung nach Ahlfeld. Arch. f. Gyn. Bd. L. 1896. Das Weib in seiner geschlechtlichen Eigenart. Nach einem Vortrage. Berlin. 
Julius Springer. I. Aufl. 1897. V. Aufl. 1904. Das Weib in seiner Geschlechtsindividualität. Ein Wort zu der Entgegnung auf meinen Vortrag von Anna L. Deutsche med. Wochenschr. 1897. No. 13. Die neue Universitätsfrauenklinik zu Göttingen. Klin, Jahrbuch. 1898.

Bd. VI. Männlich $\beta$ und weibliche Frauenheilkunde. Festrede zur Akadem. Preisverteilung in Göttingen am 5. VI. 1899. (Dieterichsche Univ.-Buchdruckerei.) Warum hilft sich die Frau nicht selbst auf dem Gebiet der Gynäkologie ? Göttingen 1899. Lehrbuch der Gynäkologie. I. Aufl. 1901. III. Aufl. 1907. Ein Wort zur Hebammenreform. Monatsschr. f. Geb. u. Gyn. 1903.

Bd. XVI. H. 3. Ahlfeld und seine intrauterine Atmung. Arch. f. Gyn. 1904. Bd. 75. H. 1. Hebammenlehrbuch (Anzeige). Zentralbl. für Gyn. 1904. No. 44. Das neue Hebammenlehrbuch. Deutsche med. Wochenschr. 1904. No. 45. Das neue Hebammenlehrbuch. 2. Mitteilung. Deutsche med. Wochenschr.

1905. No. 7. Im Auftrag des Ministeriums: Hebammenlehrbxich. 1904. II. Ausgabe 1905. Berlin. Julius Springer. Der Krebs der Gebärmutter. Ein Mahnwort an die Frauenwelt. Nach einem

in Göttingen gehaltenen Vortrage. Berlin. Julius Springer. 1905. Die Heilung des Krebses der Gebärmutter. Therap. d. Gegenwart. 1905.

No. 6. Rissmann und das Preussische Hebammenlehrbuch, Ausgabe 1905. Zeitschr. f. Medizinalbeamte. 1906. No. 7. Adolf Gusserow, Nachruf. Zentralbl. f. Gyn. Beilage. 1906. No. 8. Vortrag: Die Anzeigepflicht, ein Mittel zur Bekämpfung des Kindbettfiebers, Zeitschr. f. Medizinalbeamte. Beilage 1907. No. 1. Berichte über

Versammlungen. Ventrale Totalexstirpation des gebärenden Uterus bei Placenta praevia mit unstillbarer Blutung. Zentralbl. f. Gyn. 1909. No. 31.

Druckfehler-Berichtigung.

In der Arbeit von B. S. Schultze muss es auf S. 148 Zeile 17 von oben 15000 (statt 1300)

heissen.

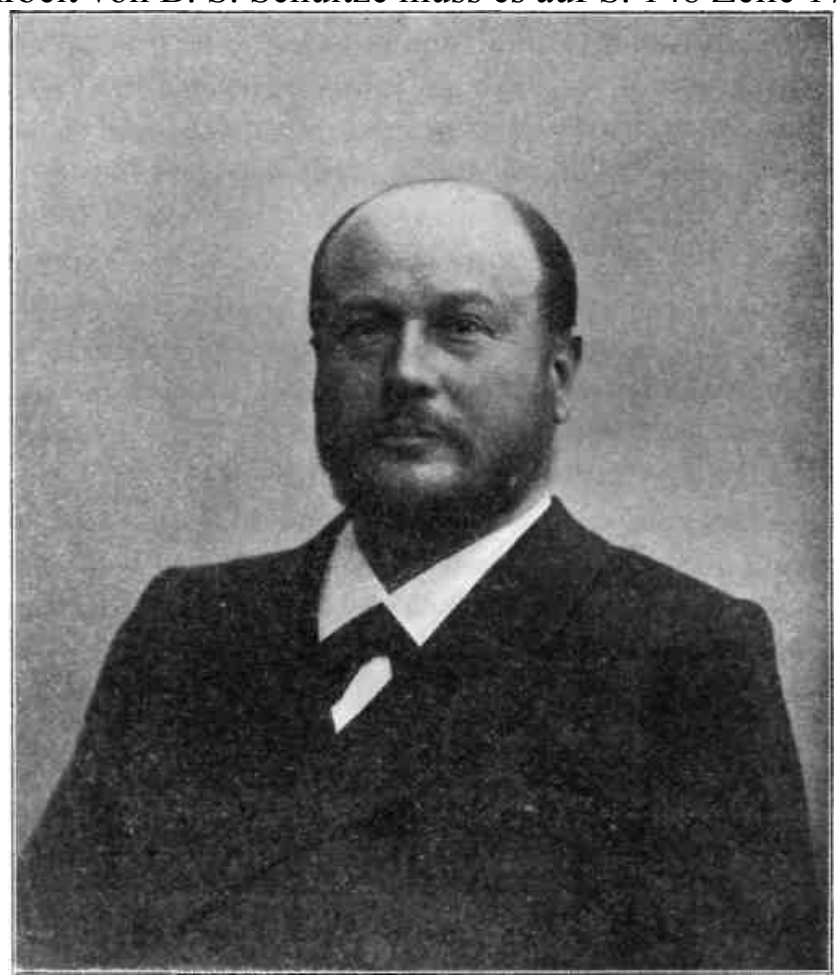

